\title{
ALUMINUM EFFECT ON SLOW AXONAL TRANSPORT: A NOVEL IMPAIRMENT OF NEUROFILAMENT TRANSPORT ${ }^{1}$
}

\author{
A. BIZZI, R. CLARK CRANE, L. AUTILIO-GAMBETTI, AND P. GAMBETTI ${ }^{2}$ \\ Division of Neuropathology, Institute of Pathology, Case Western Reserve University, Cleveland, Ohio 44106
}

Received July 5, 1983; Accepted August 19, 1983

\begin{abstract}
Administration of aluminum ( $\mathrm{Al}$ ) produces accumulation of neurofilaments (NF), called neurofibrillary tangles (NFT), in neuronal cell bodies and proximal axonal segments. This study was undertaken to investigate whether these changes are associated with impairment of the slow axonal transport.

Local administration of $\mathrm{AlCl}_{3}$ induced the formation of $\mathrm{NFT}$ in 90 to $100 \%$ of the rabbit hypoglossal neurons. ${ }^{35}$ S]Methionine was then administered to the hypoglossal nerve nuclei. The hypoglossal nerves were processed 18 or 28 days later for one- and two-dimensional SDS-polyacrylamide gel electrophoresis and fluorography. Labeled NF polypeptides and a polypeptide of 57 kilodaltons $(\mathrm{Kd})$ were not detectable beyond the proximal 9-mm segment of the hypoglossal nerve in Al-treated rabbits 18 days after labeling, whereas they were present up to $27 \mathrm{~mm}$ from the medulla in controls. Tubulin and polypeptides migrating with slow component $b$ were not significantly affected. In rabbits sacrificed 28 days after labeling, accumulation of NF subunits within the proximal $9 \mathrm{~mm}$ of hypoglossal nerve was less dramatic, and labeled NF were present up to $30 \mathrm{~mm}$ from the medulla whereas they were detectable up to $45 \mathrm{~mm}$ in controls. Morphological studies demonstrated the presence of enlarged axons filled with NF in the proximal $9 \mathrm{~mm}$ of the hypoglossal nerve. In nerve segments immediately distal, axons were markedly reduced in size and contained no NF but an apparently normal number of microtubules and other organeiles.

Transport of NF and of a 57-Kd polypeptide is markedly but reversibly slowed down or blocked within the proximal 9-mm segments of the hypoglossal nerve following $\mathrm{Al}$ administration to the hypoglossal nucleus. It is suggested that NF transport is maintained distally, resulting in lack of $\mathrm{NF}$ in axonal segments immediately distal to the block. Local Al intoxication provides a novel model of impairment of NF transport.
\end{abstract}

Neurofilaments (NF) are synthesized and assembled in the cell body of neurons and then routed to the axon where they migrate distally with the slow component $a$ (SCa) of the axonal transport (Black and Lasek, 1980; Norton and Goldman, 1980). These events appear to be affected in a variety of human pathological conditions, resulting in the accumulation of morphologically normal $\mathrm{NF}$ in neuronal cell bodies and axons (Norton and Goldman, 1980). This change is particularly prominent in giant axonal neuropathies and in amyotrophic lateral sclerosis (ALS). In giant axon neuropathies, a group of diseases including toxic as well as inherited conditions, large masses of NF distend distal segments of central and peripheral axons (Asbury, 1979; Asbury and Brown,

${ }^{1}$ This work was supported by National Institutes of Health Grants AG 00795 and NS 06239. We wish to thank Ms. Johnstone, Cole, and Krause and Mr. Sindely for their technical assistance.

${ }^{2}$ To whom correspondence should be addressed.
1980; Spencer and Schaumburg, 1976, 1977; Griffiths et al., 1981; Jones and Cavanagh, 1982). In early stages of ALS, NF instead accumulate in the cell bodies and in the proximal segments of the intraparenchymal axons of lower motor neurons (Hirano, 1982; Hirano et al., 1983).

Experimental models have added to our current understanding of some of the pathological conditions preferentially affecting NF and have also generated new information on the organization and transport of cytoskeletal components. We recently found that the rate of transport of $\mathrm{NF}$ and of two other minor polypeptide components of the SCa is selectively accelerated in 2,5hexanedione (2,5-HD) intoxication, an experimental model of giant axonal neuropathy; this is the first experimental model in which NF transport is selectively accelerated (Monaco et al., 1983).

No true experimental model is currently available for ALS. However, several investigators have pointed out the similarity between the early neuronal changes in 
ALS and those experimentally produced by either $\beta, \beta^{\prime}-$ iminodipropionitrile (IDPN) or aluminum (Al) (Bugiani and Ghetti, 1982; Troncoso et al., 1982). Systemic administration of IDPN causes focal enlargements in the intraparenchymal and proximal 5 - to $10-\mathrm{mm}$ root segments of the axons of the lower motor neurons (Chou and Hartmann, 1964, 1965; Chou and Klein, 1972; Griffin and Price, 1980). Distally, there is segregation of NF from microtubules (MT); NF are displaced to the periphery of the axon, while MT, smooth endoplasmic reticulum, and mitochondria are displaced to the center (Papasozomenos et al., 1981). It has been proposed that IDPN disrupts the NF-MT meshwork, resulting in impairment of NF transport and NF accumulation in the proximal segment of the axon (Griffin et al., 1978, 1983). A similar mechanism could be postulated for the NF accumulation observed in ALS. However, a major difference in the pathology of IDPN and that of early stages of ALS is that, in the latter, NF also accumulate in the neuronal cell bodies whereas this change is not prominent following IDPN administration (Griffin and Price, 1980). Moreover, no segregation of NF from MT and other axonal organelles has ever been reported in peripheral nerves from cases of ALS (Bradley et al., 1983).

Neuronal changes observed after $\mathrm{Al}$ administration to the central nervous system perhaps resemble more closely those in the early stages of ALS, because they include accumulation of NF in both the perikaryon and proximal axonal enlargements (Hirano, 1982; Hirano et al., 1983). The events that lead to NF accumulation following $\mathrm{Al}$ administration are unknown. A variety of metabolic abnormalities, such as increased synthesis, decreased degradation, or a primary effect on other elements of the neuronal cytoskeleton with secondary involvement of NF, may lead to the accumulation of NF. However, impairment of the axonal transport has been suggested as the most likely pathogenetic mechanism of $\mathrm{NF}$ accumulation in $\mathrm{Al}$ intoxication (Bugiani and Ghetti, 1982; Troncoso et al., 1982).

In the present study slow axonal transport and axonal morphology were investigated in the rabbit hypoglossal system following local administration of $\mathrm{Al}$ to the hypoglossal nucleus. The data obtained indicate that transport of NF and of a polypeptide of 57 kilodaltons (Kd) are markedly slowed down or blocked at the proximal 6to 9-mm segment of the hypoglossal nerve. Part of these findings has been reported (Bizzi et al., 1983).

\section{Materials and Methods}

Administration of $A l$ and $\left[{ }^{35} S J\right.$ methionine. Albino rabbits (2200 to $2900 \mathrm{gm}$ ) were anesthetized with halothane and the floor of the IVth ventricle was exposed. A solution of $\mathrm{AlCl}_{3}$ (1.5\% in saline, final pH 3.5) was injected intraparenchymally, 0.5 to $1.0 \mathrm{~mm}$ deep, in four sites along the midline at the level of the hypoglossal cranial nerve nuclei. Injections were repeated 1 week later. Controls were likewise injected with saline brought to $\mathrm{pH}$ 3.5 .

One week after the second administration of $\mathrm{Al}$ or saline, animals received $8 \mu \mathrm{l}$ of saline containing 1000 $\mu \mathrm{Ci}$ of L- $\left[{ }^{35} \mathrm{~S}\right]$ methionine $(>800 \mathrm{Ci} / \mathrm{mmol}$, New England
Nuclear, Boston, MA), divided in two series of four injections, 0.5 to $1.0 \mathrm{~mm}$ deep, within the entire length of both hypoglossal nuclei.

Eighteen or 28 days after administration of the labeled precursor, animals were perfused for 5 min with saline; the hypoglossal cranial roots and nerves were dissected out and cut in 3-mm segments using a gel slicer (Mickle Laboratory Engineering Co., Surrey, England), and the epineurium was removed under a dissecting microscope.

In an additional experiment $\left[{ }^{35} \mathrm{~S}\right]$ methionine was injected 10 days before the first administration of $\mathrm{Al}$, followed by a second $\mathrm{Al}$ administration 1 week later. The animal was sacrificed 28 days after the injection of labeled precursor, and the nerves were processed as above.

Gel electrophoresis and fluorography. Each hypoglossal nerve segment was homogenized in a microhomogenizer (Micro-metric Instrument Co., Cleveland, $\mathrm{OH}$ ) with 250 $\mu \mathrm{l}$ of chloroform:methanol (2:1); $50 \mu \mathrm{l}$ of $\mathrm{H}_{2} \mathrm{O}$ were added and vortexed. After addition of $200 \mu \mathrm{l}$ of methanol, the homogenizers were centrifuged at $1000 \times g$ for $10 \mathrm{~min}$. The supernatants were discarded, and the pellets were dissolved by homogenization with $2 \%$ SDS in $50 \mathrm{mM}$ Tris, $\mathrm{pH}$ 6.8. Radioactivity was determined in an aliquot, and the remainder was used for one- and two-dimensional SDS-polyacrylamide gel electrophoresis (1-DGE and 2-DGE), carried out as previously described (AutilioGambetti et al., 1981). All slab gels were $10 \%$ acrylamide. Fluorography was done according to the procedure of Bonner and Laskey (1974).

Morphological studies. Brain stems from all of the animals, including two sacrificed 1 week following the second Al administration, were dissected out, fixed by immersion in $10 \%$ buffered formalin, and embedded in paraffin. From the Al-treated animals and controls used for fluorography and sacrificed 18 days following injection of $\left[{ }^{35} \mathrm{~S}\right]$ methionine, one of every 10 or 20 sections was stained with the Bodian silver method (Gambetti et al., 1981); total number of neurons and percentage of neurons with Al-induced neurofibrillary tangles (NFT) were determined in both hypoglossal nerve nuclei.

For electron microscopic studies, two Al-treated and two control rabbits were fixed by perfusion through the ascending aorta with $3.5 \%$ glutaraldehyde in $2.5 \mathrm{mM}$ cacodylate buffer, $10 \mu \mathrm{M} \mathrm{MgCl} 2$ (pH 7.3), postfixed in $2 \%$ osmic acid, dehydrated in graded ethanols, and embedded in Epon. Sections were examined with a JEOL CX100 electron microscope.

\section{Results}

Neurofibrillary tangles. Morphometric analysis showed that in rabbits sacrificed 32 days following the first $\mathrm{Al}$ administration, 90 to $100 \%$ of the hypoglossal neurons contained NFT (Fig. 1). The total number of neurons in the Al-treated animals was not decreased when compared to controls. In both Al-treated and control rabbits, there were occasional small areas of necrosis and "shrunken neurons" in the midline at the point of injection. Interestingly, virtually no changes were seen in the neurons of the motor nucelus of the vagus, which were supposedly exposed to the same amount of Al. Although no quantitative study in serial sections was done, virtually all hypoglossal neurons contained NFT also in rabbits sac- 

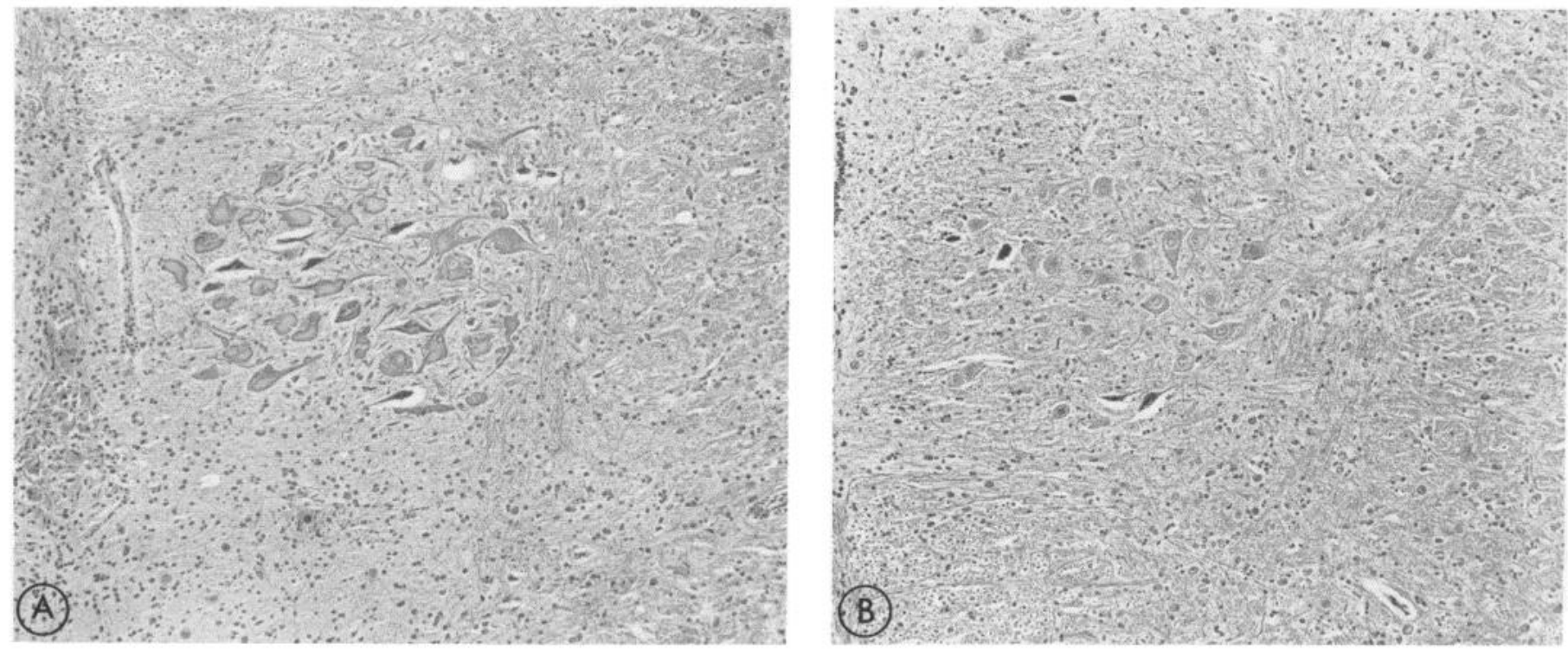

Figure 1. Cross-section of the medulla at the level of the nucleus of the hypoglossal nerve. $A$, Thirty-two days following the first $\mathrm{Al}$ administration. $B$, Control. In $A$, virtually all of the hypoglossal neurons contain prominent neurofibrillary tangles in their cell bodies. Neuronal processes are enlarged. Occasional shrunken neurons are present in Al and control hypoglossal nuclei. Paraffin embedding. Bodian silver stain. Magnification $\times 80$.

rificed 1 week following the second injection of $\mathrm{Al}$, i.e., at the time of administration of labeled methionine.

Distribution of radioactivity migrating with the slow axonal transport. The distribution of radioactivity along the hypoglossal nerve was quite different in experimental and control animals 18 days following $\left[{ }^{35} \mathrm{~S}\right]$ methionine administration (Fig. 2). In the proximal $9 \mathrm{~mm}$ of the $\mathrm{Al}$ nerves, there was a peak of radioactivity followed by a slope which was not present in the control nerves. In the first two segments of the $\mathrm{Al}$ nerve, radioactivity was significantly higher than in the control (Fig. 2). More distally, the experimental and control distributions were similar.

Fluorographic analysis of slow axonal transport. Fluorograms of consecutive segments of the hypoglossal nerve from experimental animals 18 days after injection of $\left[{ }^{35}\right.$ S]methionine (32 days following the first $\mathrm{Al}$ administration) showed that the three NF subunits were not transported beyond the proximal $9 \mathrm{~mm}$ (Fig. $3 A$ ). In controls, labeled NF subunits were present up to $27 \mathrm{~mm}$ from the medulla (Fig. $3 B$ ). The transport of another polypeptide of approximately $57 \mathrm{Kd}$ was affected in a way similar to that of the NF. Tubulin, the other major component of the $\mathrm{SCa}$, as well as polypeptides migrating with the slow component $b(\mathrm{SCb})$, were transported at rates which, in our system, were not significantly different from those of the control nerve.

In animals sacrificed 28 days after labeling ( 42 days after the first $\mathrm{Al}$ administration), the proximal accumulation of NF was less dramatic, and labeled NF subunits were present up to $30 \mathrm{~mm}$ from the medulla (Fig. $4 A$ ). In control nerves, NF subunits were detectable up to 45 $\mathrm{mm}$ from the medulla (Fig. $4 B$ ). Also, in these animals, the 57-Kd polypeptide showed a distribution similar to the NF. The nature of the 57-Kd polypeptide was further investigated with 2 -DGE and fluorography of the proximal nerve segments of Al-treated animals. A distinct

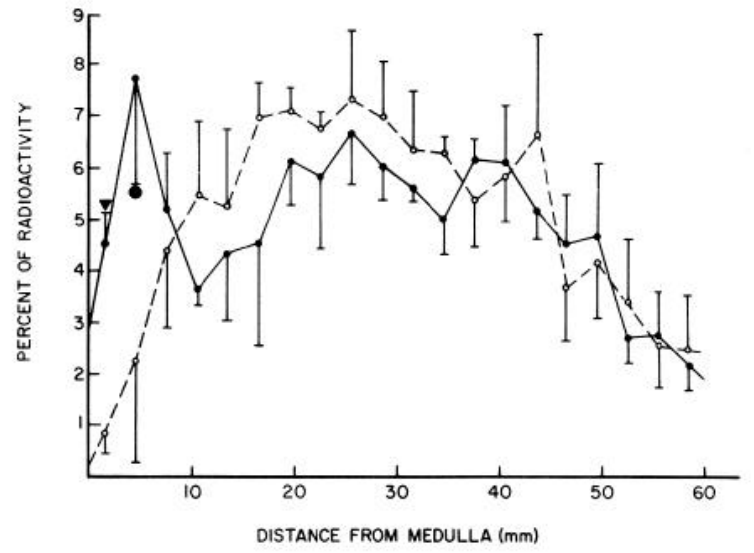

Figure 2. Distribution of the radioactivity migrating with the slow axonal transport in the hypoglossal nerve 18 days following administration of $\left[{ }^{35} \mathrm{~S}\right]$ methionine.,- Al treated, ---, controls. Radioactivity values in the first two segments of Altreated animals are significantly higher than in controls; they are not significantly different in the more distal segments. $\nabla$, $p 0.005 ; \bullet, p 0.05$.

radioactive polypeptide of $57 \mathrm{Kd}$ and a pI of 5.6 was present immediately above $\alpha$-tubulin; this polypeptide was easily seen in these fluorograms, since most of the wave of labeled tubulin had already moved distally (Fig. $5 B$ ). In control nerves, however, if the $57 \mathrm{Kd}$ is coherently transported with tubulin, the label in the latter obscures its presence. This polypeptide was similar in molecular weight and isoelectric point to a polypeptide present in NF fractions that we have previously reported to react with antibodies raised to the individual NF subunits (Autilio-Gambetti et al., 1981). To confirm their identity, an NF fraction from a normal rabbit hypoglossal nerve was added to a labeled nerve sample before 2-DGE. The radioactive polypeptide was found to co-migrate 

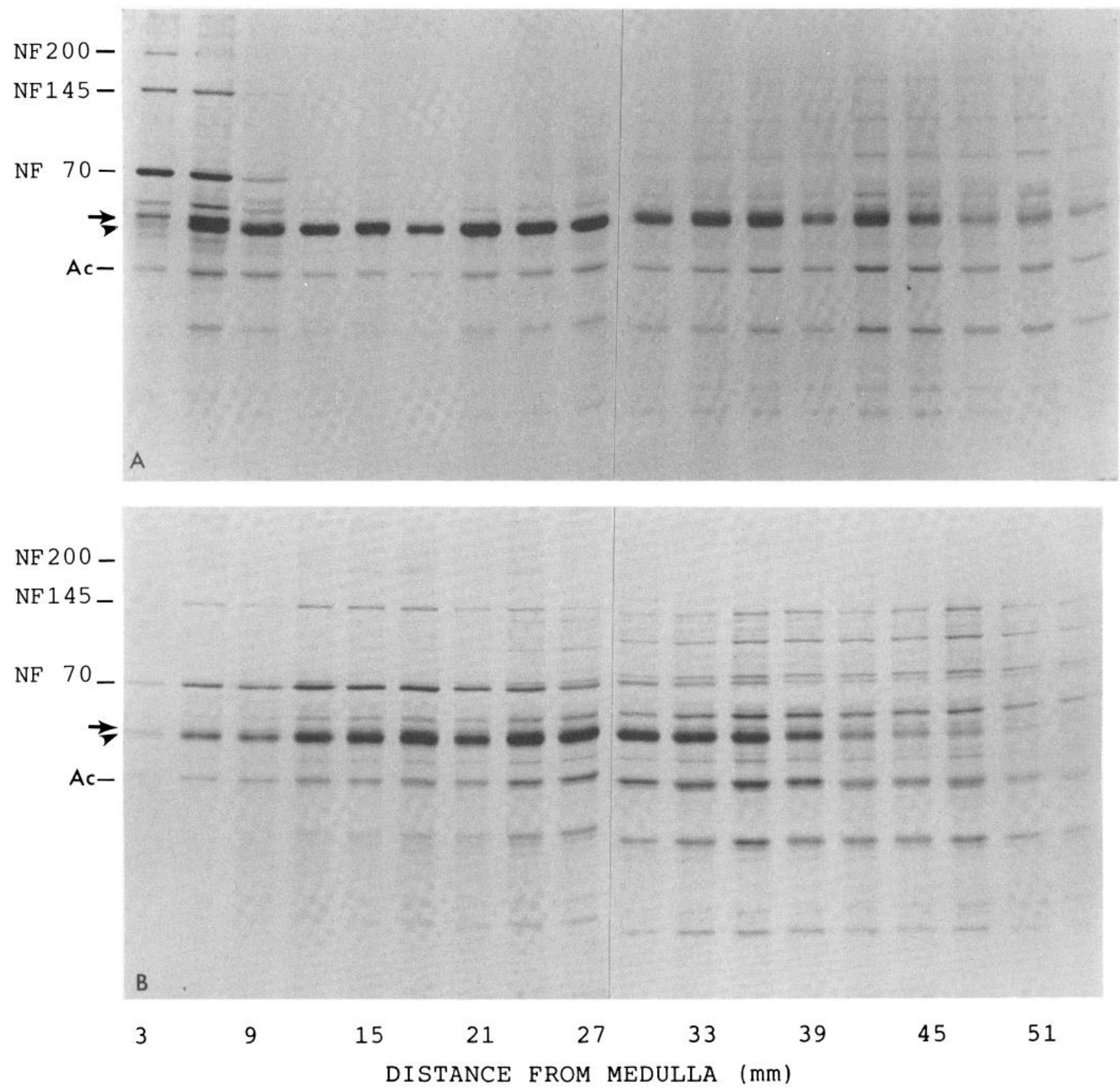

Figure 3. 1-DGE fluorograms of consecutive 3-mm segments of the hypoglossal nerve 18 days following administration of $\left[{ }^{35} \mathrm{~S}\right]$ methionine. $A$, Al treated. $B$, Control. In $A$, the neurofilament subunits $(N F 200, N F 145, N F 70)$ are seen only up to the third segment, $9 \mathrm{~mm}$ from the medulla, whereas in the control they are detectable up to $25 \mathrm{~mm}$ from the medulla. A 57 -Kd polypeptide (arrow) is prominent in the first two segments of the experimental nerve. The same polypeptide is barely detectable in the second and third segments of the control. The distribution of this polypeptide in the first segments of $\mathrm{Al}$ and control nerves suggests that it migrates with NF but that it is obscured by tubulin in the control. There is no detectable delay in the transport of tubulin (arrowhead), actin (Ac), or the polypeptides of SCb.

with the Coomassie blue-stained polypeptide present in the NF fraction.

In experiments in which $\mathrm{Al}$ was first administered 10 days following $\left[{ }^{35} \mathrm{~S}\right]$ methionine injection, no impairment of the transport of NF was seen (results not shown).

Morphology of hypoglossal nerve axons. In animals sacrificed 32 days following the first $\mathrm{Al}$ administration, numerous axons were enlarged in the proximal 6- to 9$\mathrm{mm}$ nerve segment, where fluorographic analysis showed impairment of the NF transport (Fig. 6A). Axons in segments 15 to $18 \mathrm{~mm}$ from the medulla, which had the lowest radioactivity in the distribution curve and in corresponding fluorograms showed no radioactive bands in the region above $M_{\mathrm{r}}=60,000$, were markedly reduced in size (Fig. 6B).

Electron microscopy demonstrated that the enlarged axons were filled with morphologically normal NF (Fig. 7). Among the NF there were domains containing 3 to 

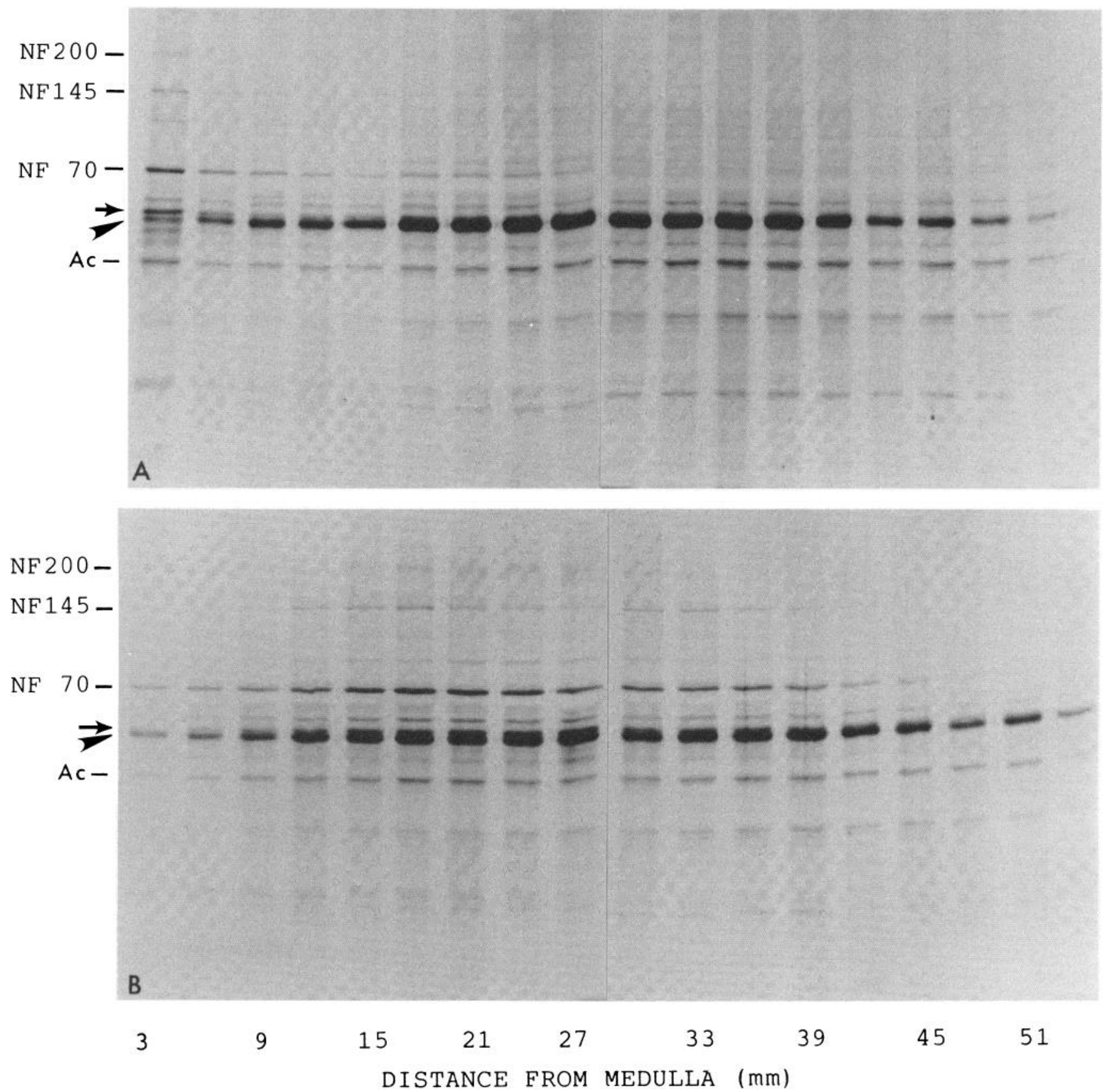

Figure 4. 1-DGE fluorograms of consecutive 3-mm segments of hypoglossal nerve, 28 days following administration of [ $\left.{ }^{35} \mathrm{~S}\right]$ methionine. $A, \mathrm{Al}$ treated. $B$, Control. There is evidence of some accumulation of NF subunits $(N F 200, N F 145, N F 70)$ in the first segment of the experimental nerve. NF subunits can be detected up to $15 \mathrm{~mm}$ from the medulla, whereas in the control they are present up to $48 \mathrm{~mm}$ from the medulla. Also, the $57-\mathrm{Kd}$ polypeptide (arrow) is prominent in the first segment of the Al nerve. Transport of SCb polypeptides appears slightly retarded in the Al nerve. Arrowhead, tubulin; Ac, actin.

30 microtubules and often axonal organelles, such as mitochondria and vesicles of smooth endoplasmic reticulum. The myelin sheaths of these axons were abnormally thin. On the contrary, the atrophic axons in the more distal segments contained only few, if any, NF, whereas the number of MT appeared to be normal (Fig. 8). The myelin sheaths of the shrunken axons were thickened. In nerve segments more than $23 \mathrm{~mm}$ from the medulla, axonal size and cytoskeleton appeared normal.

\section{Discussion}

Model of Al intoxication. The most common mode of $\mathrm{Al}$ administration is by intracisternal injection. When administered by this route to receptive animals, $\mathrm{Al}$ induces widespread neurofibrillary changes in the CNS (Klatzo et al., 1965; Terry and Peña, 1965; Crapper and Dalton, 1973; Wisniewski et al., 1980b; Bugiani and Ghetti, 1982; Troncoso et al., 1982). However, adult 


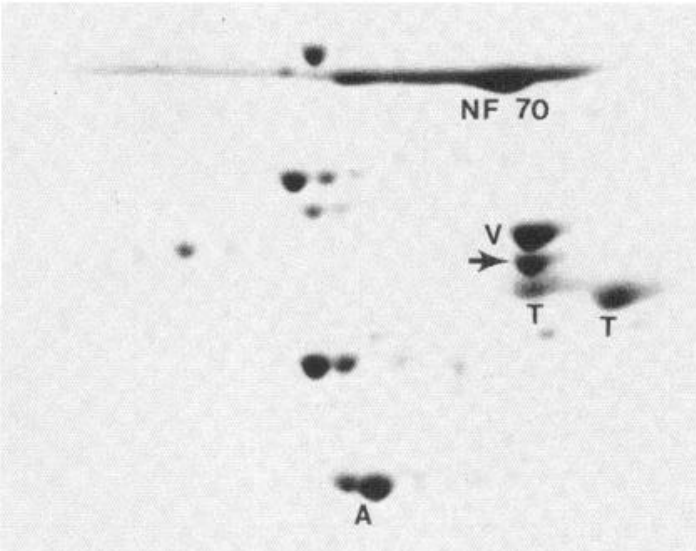

A

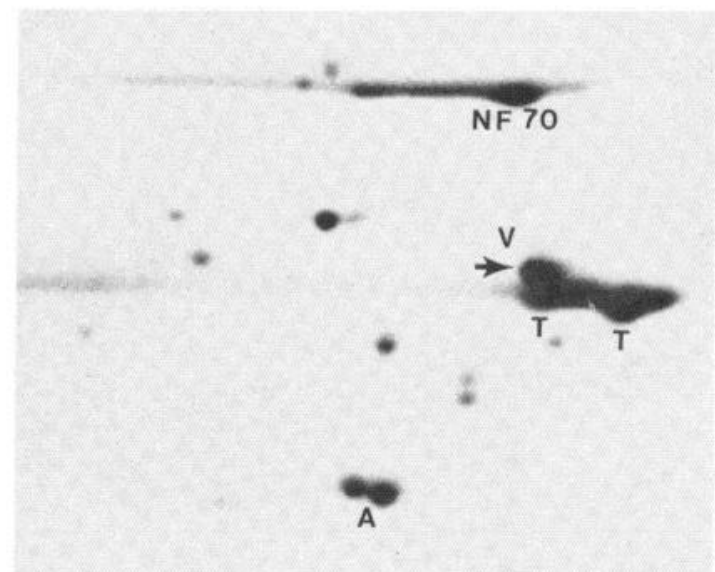

B

Figure 5. 2-DGE of the proximal segments of a nerve from an Al-treated animal 18 days following administration of $\left[{ }^{35} \mathrm{~S}\right]$ methionine. An NF fraction prepared from a normal, nonradioactive rabbit (Autilio-Gambetti et al., 1981) was added to the nerve sample before electrophoresis. $A$, Coomassie stained. $B$, Corresponding fluorogram. Note the heavy labeling of the $57-\mathrm{Kd}$ polypeptide (arrow) and the absence of radioactivity in vimentin ( $V)$. $N F 70,70$-Kd neurofilament subunit; $T$, tubulin; $A$, actin. In this gel NF 70 focused at a more basic pI than usual.
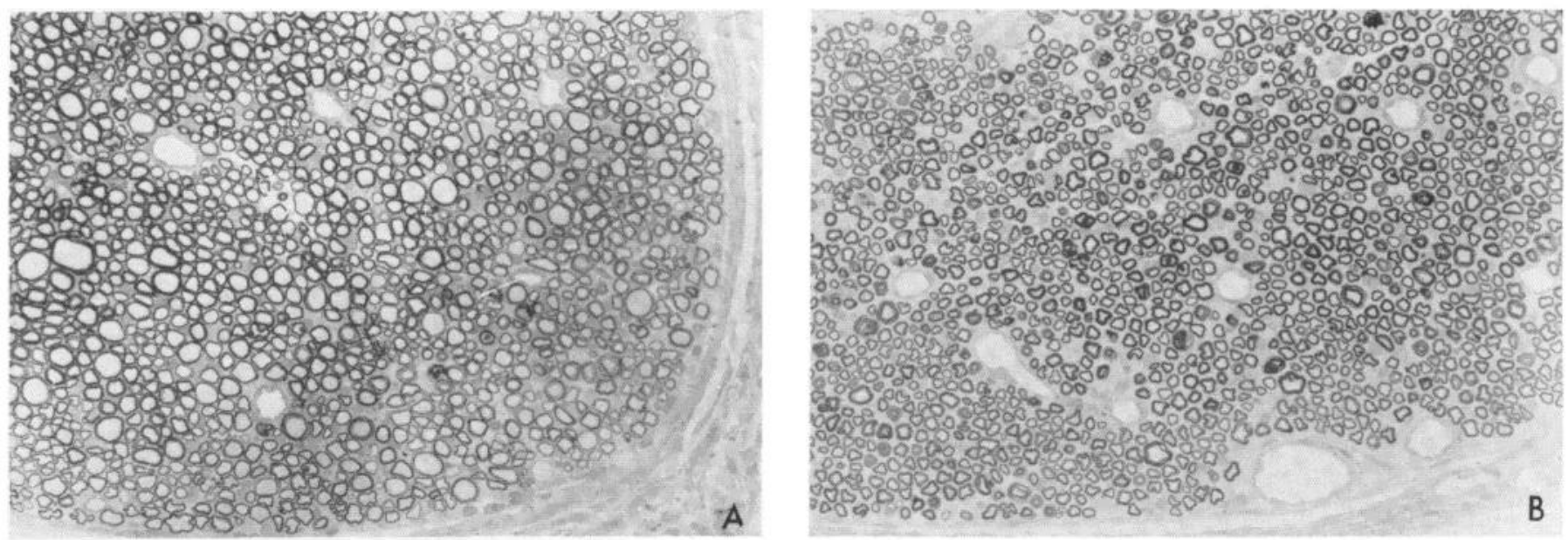

Figure 6. Light micrographs of cross-sections from the hypoglossal nerve of a rabbit 32 days following the first Al administration. $A$, Three to $6 \mathrm{~mm}$ from the medulla. $B$, Twelve to $15 \mathrm{~mm}$ from the medulla. Several axons are enlarged and surrounded by a thin myelin sheath in the segment 3 to $6 \mathrm{~mm}$ from the medulla $(A)$, corresponding to the site of slowdown or block of neurofilament subunits shown by fluorography. On the contrary, 12 to $15 \mathrm{~mm}$ from the medulla $(B)$ axons are markedly reduced in size and are often surrounded by a thick myelin. Epon embedding. Toluidin blue staining. Magnification $\times 250$.

animals commonly develop severe neurological signs and recurrent seizures and usually die within 1 to 3 weeks (Wisniewski and Terry, 1970; Yates et al., 1976). A chronic model was obtained by subarachnoid injection of a slurry of $\mathrm{Al}$ powder (Bugiani and Ghetti, 1982; Wisniewski et al., 1982). In this model, animals display severe and generalized neurological dysfunction, and NFT are present in 40 to $60 \%$ of the anterior horn neurons (Bugiani and Ghetti, 1982). Recently, Kosik et al. (1983) produced NFT in more than $80 \%$ of the anterior horn cells of the lumbar region of the spinal cord of rabbits by multiple local injections. With local administration of $\mathrm{Al}$, we have now induced the formation of NFT in 90 to $100 \%$ of the neurons in both hypoglossal nuclei with no significant cell loss. During the entire period of intoxi- cation the animals remained free of major neurological symptoms. These findings and the proven suitability of the hypoglossal system for the study of axonal transport indicate that our model can be successfully used to analyze the effect of chronic $\mathrm{Al}$ intoxication on slow axonal transport.

Al effect on slow axonal transport. Both fluorographic and morphological data show that, following $\mathrm{Al}$ administration, NF polypeptides accumulate in the proximal 6 to $9 \mathrm{~mm}$ of the hypoglossal nerve axons, while they are markedly decreased in the nerve segments immediately distal to this region. More distally, however, axons are normal. These results clearly indicate that, following $\mathrm{Al}$ administration to the hypoglossal nucleus, transprt of NF is markedly slowed down or blocked within the 

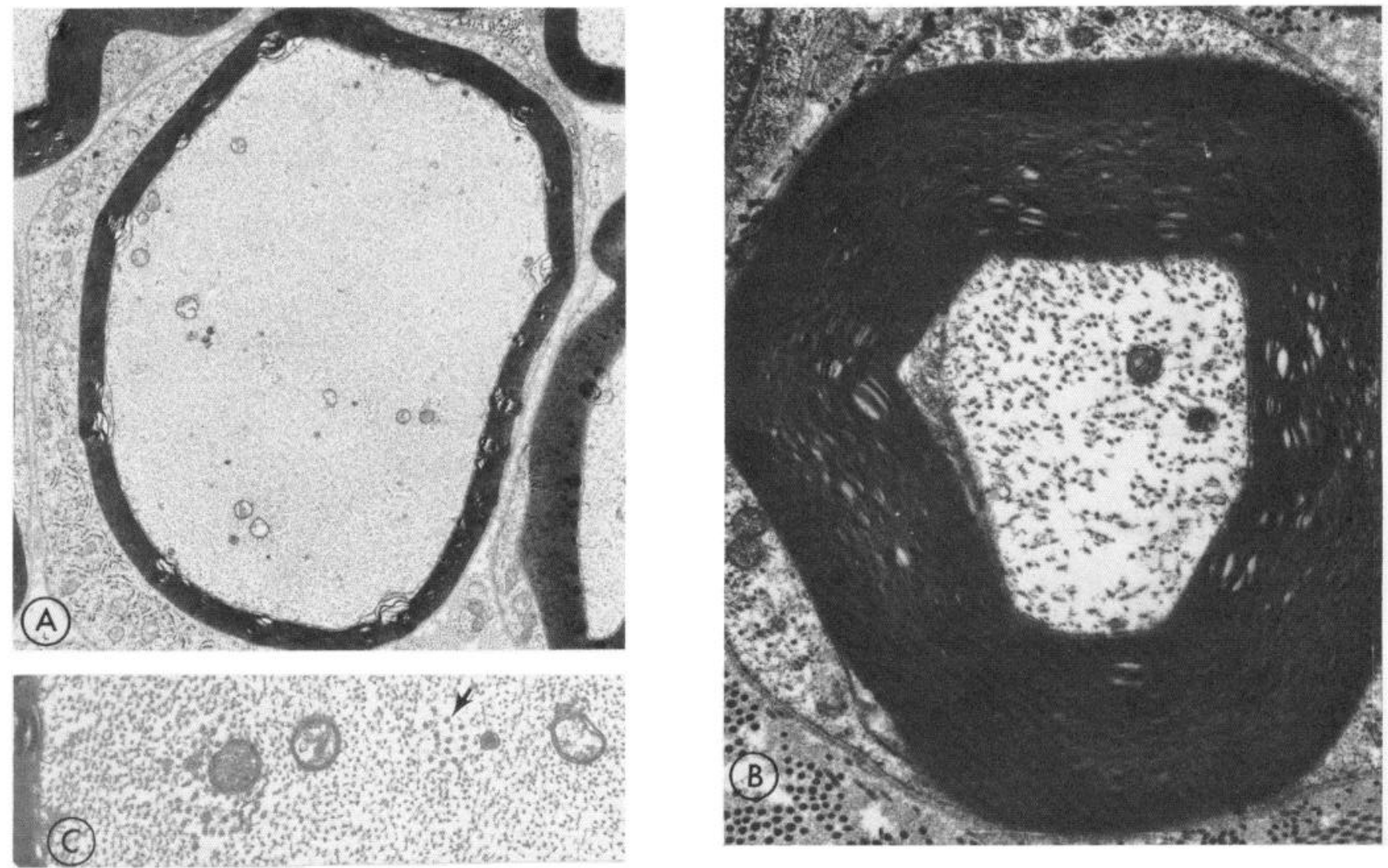

Figure 7. Electron micrographs of nerve axons from segments $A$ and $B$ of Figure 6 . The enlarged axons are filled with neurofilaments $(A)$; within the mass of neurofilaments $(C)$ there are domains of microtubules $($ arrow) and other axonal organelles such as mitochondria and cisterns of smooth endoplasmic reticulum. On the contrary, axons immediately distal $(B)$ do not contain NF but have an apparently normal number of MT and other organelles. They are also markedly reduced in size and surrounded by a thick myelin sheath. Magnifications: $A, \times 6,500 ; B, \times 17,000 ; C, \times 22,000$.

proximal 6 to $9 \mathrm{~mm}$ of the hypoglossal nerve axons. NF transport, however, is probably maintained distally. Because of this focal blockade, adjacent segments of the same axons show a proximal region loaded with NF, followed by another immediately distal lacking NF. A diagrammatic representation of the morphological changes is shown in Figure 8.

In animals sacrificed 42 rather than 32 days following the first $\mathrm{Al}$ administration, $\mathrm{NF}$ transport was still delayed, and there was evidence that accumulation of NF polypeptides in the proximal segments had occurred. However, labeled NF polypeptides were seen beyond the proximal 6- to 9-mm segments of the hypoglossal nerve. Thus, the impairment of $\mathrm{NF}$ transport produced by $\mathrm{Al}$ is reversible, with transport of NF resuming 25 to 35 days following $\mathrm{Al}$ administration. These findings are consistent with previous morphological studies that have pointed out that Al-induced NFT and intraparenchymal axonal enlargements decrease between 4 and 8 weeks after $\mathrm{Al}$ intoxication (Troncoso et al., 1982). Resumption of NF transport is probably the (or one of the) mechanism(s) responsible for the reduction in the number of NFT observed in chronically intoxicated animals (Bugiani and Ghetti, 1982; Wisniewski et al., 1980b).

Impairment of NF transport and formation of NFT. Transport of $\mathrm{NF}$ is not impaired when $\mathrm{Al}$ is administered after the labeled precursor. This finding, in conjunction with the morphological data, indicates that local administration of Al does not affect the transport of NF that have entered the slow transport system and are already beyond the 6 - to 9 -mm proximal segment of the hypoglossal nerve axons. This initial segment of the peripheral axons seems to be more vulnerable than the distal region to toxic compounds that affect NF transport, as not only $\mathrm{Al}$, but systemic administration of IDPN also produces a block of NF transport in this particular axonal segment (Griffin et al., 1978; P. Gambetti, A. Bizzi, and L. Autilio-Gambetti, unpublished data). It has recently been shown that the initial axonal segment of the Aplysia giant neuron is the site of extensive post-translational modification of NF (Drake and Lasek, 1983; P. F. Drake and R. J. Lasek, personal communication). Similar but quantitatively lesser changes also occur in the optic system of mammals during axonal transport (Nixon et al., 1982). It is tempting to suggest that in motor axons a remodeling of the cytoskeleton might take place in the intraparenchymal as well as in the proximal peripheral segments. This would explain the preferential effect of $\mathrm{Al}$ and other toxic compounds in this region.

The severe impairment of NF transport in the initial region of the peripheral axon in $\mathrm{Al}$ intoxication raises the question as to the role this impairment plays in the formation of NFT in neuronal cell bodies and intraparenchymal axons. It is difficult to explain these lesions 


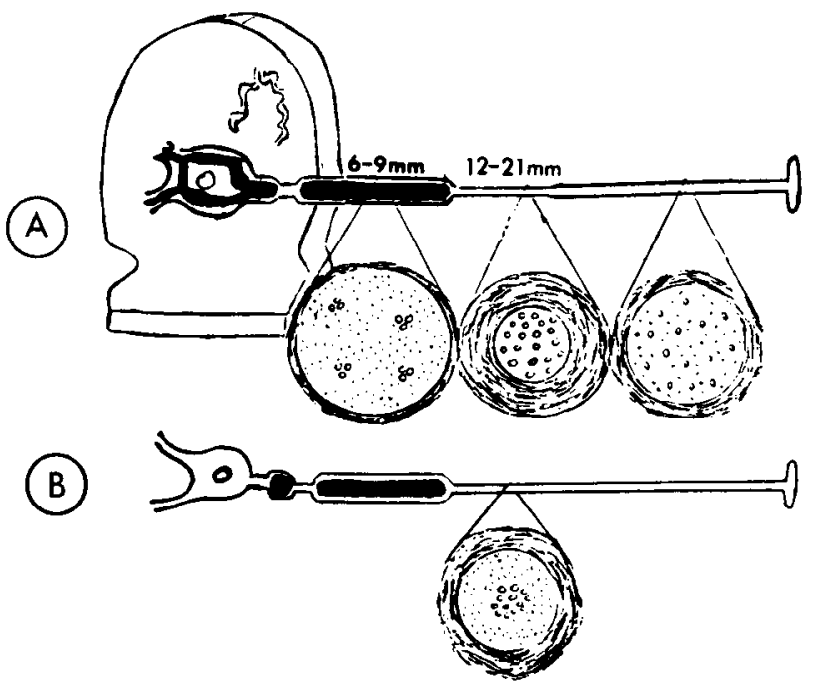

Figure 8. Diagram summarizing the morphological changes. $A$, Thirty-two days following the first $\mathrm{Al}$ administration to the hypoglossal nerve nuclei. $B$, Following systemic administration of IDPN according to Papasozomenos et al. (1981). In Al intoxication $(A), \mathrm{NF}$ accumulate in the neuronal cell body and proximal segments of the axon up to $9 \mathrm{~mm}$ from the medulla. The axonal accumulation of $\mathrm{NF}$ is not continuous. In the "IDPN axon" $(B)$ the axonal accumulation of NF is similar to that following $\mathrm{Al}$ administration. Following IDPN, however, accumulation of NF in the neuronal cell bodies is not commonly seen. The cytoskeletal organization is similar in both conditions in the enlarged region of the axon, but it is different more distally. In Al, axonal segments immediately distal to the enlarged regions lack NF, are markedly reduced in size, and are surrounded by a thick myelin sheath. More distally, axonal cytoskeletal and size are normal. In IDPN, NF are segregated at the periphery and MT clustered at the center all along the axon, distal to the enlarged segments. The size of the axons is also slightly reduced.

with a block of NF transport in the proximal nerve segments producing a damming effect with a disto-proximal accumulation of NF. In this case, axons would be continuously enlarged up to the cell body. On the contrary, the axonal enlargements in $\mathrm{Al}$ intoxication are segmentary. The presence of focal enlargements in the intraparenchymal axons suggests that transport is impaired focally, all along the proximal region of the axon up to the proximal 9-mm peripheral segment, which would represent either the initial or the last block.

This question may be addressed in simpler axonal systems that allow for the comparative study of the composition of the cytoskeleton during transport along the most proximal region of the axon or with morphological studies which may resolve the initial site(s) of NF accumulation in adult animals. These studies have to take into account that the initial NF accumulation may occur in the proximal segments of peripheral axons. The only detailed study available demonstrated that NF accumulation at the first axonal internode precedes that in the cell body (Troncoso et al., 1982). However, the proximal region of the peripheral axons was not examined in these studies.

The molecular mechanism by which $\mathrm{Al}$ impairs $\mathrm{NF}$ transport remains to be determined. Recent in vitro studies show that $\mathrm{Al}$ binds to calmodulin, resulting in a loss of its ability to interact with other proteins (Siegel and Haug, 1983). NF transport might be impaired by this binding since calmodulin with fodrin and perhaps other proteins, such as actin, are thought to be involved in the transport of cytoskeletal components (Levine and Willard, 1983; Willard, 1983; K. Heriot, P. Gambetti, and R. J. Lasek, submitted for publication).

The 57-Kd polypeptide. Block of the transport was not limited to the NF. The transport of a $57-\mathrm{Kd}$ polypeptide was also affected in a similar way to that of NF. Along with the NF, it accumulated in the proximal 6- to $9-\mathrm{mm}$ segments of the peripheral axons. The $57-\mathrm{Kd}$ polypeptide was also present in the hypoglossal nerve of normal rabbits, where our preliminary data suggest that it is transported with the SCa. Fluorography of 2-DGE confirmed that this polypeptide is distinct from $\alpha$-tubulin and is identical to a polypeptide present in NF fractions that we previously reported to immunoreact with antibodies raised to the individual NF subunits (AutilioGambetti et al., 1981). The 57-Kd polypeptide of the rabbit hypoglossal nerve is also recognized by these antibodies (data not shown). Although the nature of this polypeptide has to be investigated further, it seems unlikely that it is an artifactual product. We have recently observed that a polypeptide of $64 \mathrm{Kd}$, recognized by a monoclonal antibody directed to an epitope common to all of the intermediate filaments (Pruss and Mirsky, 1981), is transported with the SCa in the rat optic system. In 2,5-HD intoxication in which transport of $\mathrm{NF}$ is selectively accelerated, the 64-Kd polypeptide migrates with the NF (S. Monaco, L. Autilio-Gambetti, and P. Gambetti, submitted for publication). These data suggest that both the 57- and the 64-Kd polypeptides are NF related, since their rate of axonal transport parallels that of NF in normal as well as in experimental models that selectively affect NF transport. The 57-Kd polypeptide might be characteristic of the peripheral and the 64-Kd of the central axonal pathways.

Effect of $A l$ and IDPN on slow axonal transport. The effect of $\mathrm{Al}$ on the slow axonal transport differs from that of IDPN (Fig. 8). In the presence of IDPN, transport of NF is uniformly slowed down or stopped in place along the entire peripheral axon distal to the initial 5 to 10 $\mathrm{mm}$ (Griffin et al., 1978). Because of this uniform involvement of the distal part of the peripheral axon, the "longitudinal" segregation of the axonal cytoskeleton we observed in $\mathrm{Al}$ intoxication cannot occur. Moreover, IDPN axons distal to the NF-containing enlargements show segregation of NF toward the periphery and clustering of $\mathrm{MT}$ and other axonal organelles at the center (Papasozomenos et al., 1981) (Fig. 8). This "transverse" segregation was not observed in the $\mathrm{Al}$ axon. An explanation of these differences might be that IDPN is administered systemically whereas Al locally. However, the lack of significant NF accumulation in the neuronal cell body following IDPN treatment suggests that the differences between the effect of the two drugs is not based only on the different mode of administration. As a novel model of impairment of NF transport, $\mathrm{Al}$ intoxication brings to light new features of the axonal cytoskeleton. The "longitudinal" segregation shows that the block of NF transport in one region of the axon may have no effect on the transport of NF in the part of the axon 
distal to the block, suggesting that the propulsive mechanism for the migration of NF is present all along the axon. It also gives a dramatic example of the relationship between NF and the size of the axon (Friede and Samorajski, 1970; Hoffman et al., 1981). The "Al axon" also provides a new model to study the organization of the cytoskeleton and the transport in axonal segments with an excess or a lack of NF.

Al changes as a model for early neuronal changes of $A L S$. For many years $\mathrm{Al}$ intoxication was thought of as a valid model for Alzheimer's disease (Crapper et al., 1976). More recently, however, although the similarity between these two conditions has been de-emphasized (Wisniewski et al., 1980a), it has been pointed out that the Al-induced neuronal pathology shows many morphological similarities to that of ALS (Troncoso et al., 1982). As in Al intoxication, in early stages of ALS, clusters of morphologically normal NF accumulate in the neuronal cell body and intraparenchymal axons of lower motor neurons (Hirano, 1982; Hirano et al., 1983). Recently, in one case of ALS autopsied at an early stage of the disease, we have observed axonal enlargements loaded with NF in the proximal $10-\mathrm{mm}$ segments of the spinal central roots $(H$. Mitsumoto and P. Gambetti, manuscript in preparation). These changes indicate that in ALS also transport of NF is impaired. However, the "longitudinal" segregation still has to be found in ALS to prove that the type of impairment of NF transport that we have uncovered in $\mathrm{Al}$ intoxication applies also to ALS.

\section{References}

Asbury, A. K. (1979) Neuropathies with filamentous abnormalities. In Current Topics in Nerve and Muscle Research, H. A. Aguayo and G. Karpati, eds., pp. 243-254, Excerpta Medica, Amsterdam.

Asbury, A. K., and M. J. Brown (1980) The evolution of structural changes in distal axonopathies. In Experimental and Clinical Neurotoxicology, P. S. Spencer and H. H. Schaumburg, eds., pp. 179-192, Williams \& Wilkins, Baltimore.

Autilio-Gambetti, L., M. E. Velasco, J. Sipple, and P. Gambetti (1981) Immunochemical characterization of antisera to rat neurofilament subunits. J. Neurochem. 37: 1260-1265.

Bizzi, A., R. Crane, M. Yoon, L. Autilio-Gambetti, and P. Gambetti (1983) The axonal transport of neurofilaments is impaired in aluminum intoxication. J. Neuropathol. Exp. Exp. Neurol. 42: 331.

Black, M. M., and R. J. Lasek (1980) Slow components of axonal transport: Two cytoskeletal networks. J. Cell Biol. 86: 616-623.

Bonner, W. M., and R. Laskey (1974) A film detection method for tritium labelled proteins and nucleic acids in polyacrylamide gels. Eur. J. Biochem. 46: 83 . 88.

Bradley, W. G., P. Good, C. G. Rasool, and L. S. Adelman (1983) Morphometric and biochemical studies of the peripheral nervous system in amyotrophic lateral sclerosis. Ann. Neurol. 14: 267-277.

Bugiani, O., and B. Ghetti (1982) Progressing encephalomyelopathy with muscular atrophy, induced by aluminum powder. Neurobiol. Aging 3: 209-222.

Chou, S. M., and H. A. Hartmann (1964) Axonal lesions and waltzing syndrome after IDPN administration in rats. Acta Neuropathol. 3: 428-450.

Chou, S. M., and H. A. Hartmann (1965) Electron microscopy of focal neuroaxonal lesions produced by $\beta, \beta^{\prime}$-iminodipropionitrile (IDPN) in rats. Acta Neuropathol. 4: 590-603.

Chou, S. M., and R. A. Klein (1972) Autoradiographic studies of protein turnover in motoneurons of IDPN-treated rats. Acta Neuropathol. 22: 183-189.

Crapper, D. R., and A. J. Dalton (1973) Aluminum induced neurofibrillary degeneration, brain electrical activity and alterations in acquisition and retention. Physiol. Behav. 10: 935-945.

Crapper, D. R., S. S. Krishnan, and S. Quittkat (1976) Aluminum neurofibrillary degeneration and Alzheimer's disease. Brain 99: 67-80.

Drake, P. F., and R. J. Lasek (1984) Regional differences in the neuronal cytoskeleton. J. Neurosci., in press.

Friede, R. L., and T. Samorajski (1970) Axon caliber related to neurofilaments and microtubules in sciatic nerve fibers of rats and mice. Anat. Rec. 167: 379-387.

Gambetti, P., L. Autilio-Gambetti, and S. Ch. Papasozomenos (1981) Bodian's silver method stains neurofilament polypeptides. Science 213: 1521-1522.

Griffin, J. W., and D. L. Price (1980) Proximal axonopathies induced by toxic chemicals. In Experimental and Clinical Neurotoxicology, P. S. Spencer and H. H. Schaumburg, eds., pp. 161-178, Williams \& Wilkins, Baltimore.

Griffin, J. W., P. N. Hoffman, A. W. Clark, P. T. Carroll, and D. L. Price (1978) Slow axonal transport of neurofilament proteins: Impairment by $\beta, \beta^{\prime}$-iminodipropionitrile administration. Science 202: 633-635.

Griffin, J. W., K. E. Fahnestock, D. L. Price, and P. N. Hoffman (1983) Microtubule-neurofilament segregation produced by $\beta, \beta^{\prime}$-iminodipropionitrile: Evidence for the association of fast axonal transport with microtubules. J. Neurosci. 3: 557-566.

Griffiths, I. R., P. T. A. Kelly, S. Carmichael, M. McCulloch, and M. Waterston (1981) The relationship of glucose utilization and morphologic change in the visual system in hexacarbon neuropathy. Brain Res. 222: 447-451.

Hirano, A. (1982) Aspects of the ultrastructure of amyotrophic lateral sclerosis. In Human Motor Neuron Diseases, L. P. Rowland, ed., pp. 75-88, Raven Press, New York.

Hirano, A., S. Sasaki, H. Donnenfeld, and I. Nakano (1983) Neurofilamentous changes in amyotrophic lateral sclerosis (ALS). J. Neuropathol. Exp. Neurol. 42: 332A.

Hoffman, P. N., J. W. Griffin, and D. L. Price (1981) The dynamic role of the axon cytoskeleton: Regulation of axon caliber. J. Neuropathol. Exp. Neurol. 40: 316A.

Jones, H. B., and J. B. Cavanagh (1982) The early evolution of neurofilamentous accumulations due to 2,5-hexanediol in the optic pathways of the rat. Neuropathol. Appl. Neurobiol. 8: 289-301.

Klatzo, I., H. Wisniewski, and E. Streicher (1965) Experimental production of neurofibrillary degeneration. 1. Light microscopic observations. J. Neuropathol. Exp. Neurol. 24: 187199.

Kosik, K. S., W. G. Bradley, P. F. Good, C. G. Rasool, and D. J. Selkoe (1983) Cholinergic function in lumbar aluminum myelopathy. J. Neuropathol. Exp. Neurol. 42: 365-375.

Levine, J., and M. Willard (1983) Redistribution of fodrin (a component of the cortical cytoplasm) accompanying capping of cell surface molecules. Proc. Natl. Acad. Sci. U. S. A. 80: 191-195.

Monaco, S., R. Crane, L. Autilio-Gambetti, and P. Gambetti (1983) Transport of neurofilaments is accelerated in 2,5hexanedione axonopathy. J. Neuropathol. Exp. Neurol. 42: 330 .

Nixon, R. A., B. A. Brown, and C. A. Marotta (1982) Posttranslational modification of neurofilament protein during axoplasmic transport: Implications for regional specialization of CNS axons. J. Cell Biol. 94: 150-158. 
Norton, W. T., and J. E. Goldman (1980) Neurofilaments. In Proteins of the Nervous System, Ed. 2, R. A. Bradshaw and D. M. Schneider, eds., pp. 301-329, Raven Press, New York.

Papasozomenos, S. Ch., L. Autilio-Gambetti, and P. Gambetti (1981) Reorganization of axoplasmic organelles following $\beta, \beta^{\prime}$-iminodipropionitrile administration. J. Cell Biol. 91: 866-871.

Pruss, R. M., R. Mirsky, and M. C. Raff (1981) All classes of intermediate filaments share a common antigenic determinant defined by a monoclonal antibody. Cell 27: 419-428.

Siegel, N., and A. Haug (1983) Aluminum interaction with calmodulin. Evidence for altered structure and function from optical and enzymatic studies. Biochim. Biophys. Acta 744: $36-45$.

Spencer, P. S., and H. H. Schaumburg (1976) Central and peripheral distal axonopathies. Prog. Neuropathol. 3: 253295.

Spencer, P. S., and H. H. Schaumburg (1977) Ultrastructural studies in the dying back process. III. The evolution of experimental peripheral giant axonal degeneration. J. Neuropathol. Exp. Neurol. 36: 276-299.

Terry, R. D., and C. Peña (1965) Experimental production of neurofibrillary degeneration. II. Electron microscopy, phosphate histochemistry and electron probe analysis. J. Neuropathol. Exp. Neurol. 24: 200-210.

Troncoso, J. C., D. L. Price, J. W. Griffin, and I. M. Parhad
(1982) Neurofibrillary axonal pathology in aluminum intoxication. Ann. Neurol. 12: 278-283.

Willard, M. (1983) Neurofilaments and axonal transport. In Neurofilaments, C. A. Marotta, ed., pp. 86-116, University of Minnesota Press, Minneapolis.

Wisniewski, H., and R. D. 'Terry (1970) An experimental approach to the morphogenesis of neurofibrillary degeneration and the argyrophilic plaque. In Ciba Foundation Symposium on Alzheimer's Disease and Related Conditions, G. E. W. Wolstenholme and M. O'Connor, eds., pp. 223-248, Churchill, London.

Wisniewski, H. M., K. Iqbal, and J. R. McDermott (1980a) Aluminum-induced neurofibrillary changes: Its relationship to senile dementia of the Alzheimer's type. Neurotoxicology 1: $121-124$.

Wisniewski, H. M., J. A. Sturman, and J. W. Shek (1980b) Aluminum chloride induced neurofibrillary changes in the developing rabbit: A chronic animal model. Ann. Neurol. 8: 479-490.

Wisniewski, H. M., J. A. Sturman, and J. W. Shek (1982) Chronic model of neurofibrillary changes induced in mature rabbits by metallic aluminum. Neurobiol. Aging 3: 11-22.

Yates, C. M., A. Gordon, and H. Wilson (1976) Neurofibrillary degeneration induced in the rabbit by aluminum chloride: Aluminum neurofibrillary tangles. J. Neuropathol. Appl. Neurobiol. 2: 131-144. 